\title{
Les particularités pollenanalytiques de sédiments glaciaires en Oisans (Isère, France)
}

\author{
Pollenanalytical particularities of glacial sediments \\ in Oisans (Isère, France)
}

\author{
M. Coûteaux \\ Laboratoire de Botanique historique et Palynologie, E.R.A. du C.N.R.S. n ${ }^{\circ} 404$. \\ Faculté des Sciences et Techniques St-Jérôme, 13397 Marseille Cedex 13.
}

Les effets des pulsations glaciaires sur les spectres polliniques reconstitués à partir des sédiments déposés dans la région où elles se manifestent, sont complexes, en particulier du fait d'apports allochtones.

L'examen des sédiments de trois phases caractéristiques permet de le reconnaitre en Oisans. Nous étudions en premier lieu les conditions subactuelles pour tenter ensuite d'interpréter les phénomènes observés dans le Tardiglaciaire.

\section{La récurrence des $17^{\mathrm{e}}-19^{\mathrm{e}}$ siècles} de notre ère

En Oisans, durant l'Holocène, les diagrammes polliniques ne permettent pas de déceler de récurrences glaciaires, sauf à la fin du Subatlantique. Les traces géomorphologiques de cet englacement sont évidentes et abondantes. Mais les diagrammes polliniques sont d'interprétation complexe, car l'avancée glaciaire ne pénètre pas de régions forestières. En outre, l'influence humaine sur les formations végétales est forte, même à haute altitude, avant et après la période de récurrence (Coûteaux, 1983c).

L'étude de trois sites de la vallée de La Lavey, privilégiés par leur proximité de fronts glaciaires, permet l'interprétation d'ensembles polliniques assez paradoxaux. Selon l'étage de végétation, selon le sédiment analysé et selon la position du site étudié par rapport au glacier, les diagrammes polliniques donnent des images très différentes de l'influence de la récurrence glaciaire sur la végétation.

\begin{abstract}
1.1. Interfluve du glacier du lac des Bèches et du glacier d'Entre-Pierroux (fig. 1)

Une petite tourbière $(2380 \mathrm{~m})$ est située à l'abri des épandages proglaciaires (fig. 2) à $800 \mathrm{~m}$ des fronts actuels du glacier du Lac des Bèches et du glacier d'EntrePierroux.
\end{abstract}

La courbe de Picea est quasi continue dès $23 \mathrm{~cm}$ (second millénaire de notre ère : Coûteaux, 1982 d). Les quatre $\mathrm{cm}$ supérieurs, caractérisés par une augmentation des fréquences polliniques de Pinus et Picea, témoignent de la situation subactuelle $\left(19^{\mathrm{c}}\right.$ et $20^{\mathrm{c}}$ siècles).

Les secteurs du vallon de La Lavey d'altitude supérieure à $2000 \mathrm{~m}$ sont situés au-dessus de la limite forestière dès la fin de l'Atlantique (Coûteaux, 1984), les dernières destructions des îlots boisés les plus élevés se situent au début du second millénaire de notre ère (Coûteaux, $1983 \mathrm{c}$ ). La pression anthropique la plus forte a précédé la récurrence glaciaire des $17^{c}-19^{e}$ siècles, mais l'altitude élevée du site explique l'absence de manifestation pollinique locale d'anthropisation, contrairement aux tourbières moins élevées. Or, l'analyse de toutes les tourbières subatlantiques (dans la vallée de La Lavey) prouve que les sédiments des périodes à pression anthropique modeste déposés entre les $15^{\mathrm{c}}$ et $19^{\mathrm{c}}$ siècles, sont paradoxalement caractérisés par des fréquences polliniques très faibles de Pinus, qui ne résultent donc pas de déboisements anthropiques. De plus, la récurrence glaciaire n'a atteint aucune zone forestière.

Tout permet de penser que la dernière récurrence glaciaire se manifeste dans ce diagramme par les spectres de 11 à $5 \mathrm{~cm}$, caractérisés par les fréquences polliniques les plus basses de Pinus.

Les conditions climatiques favorables à l'extension glaciaire, ont donc été défavorables à la vitalité de Pinus, induisant une diminution de la productivité pollinique.

Il est possible que la fertilité de Picea abies et de Alnus viridis ait été également amoindrie. 
Figure 1 - Pelouse, mares et tourbières $(2380 \mathrm{~m})$ à l'interfluve des vallons du glacier d'Entre-Pierroux (A), du glacier du Lac des Bèches (B). C : prélèvement pollinique, voir $\S 1.1$ (Cliché 10/9/1982).
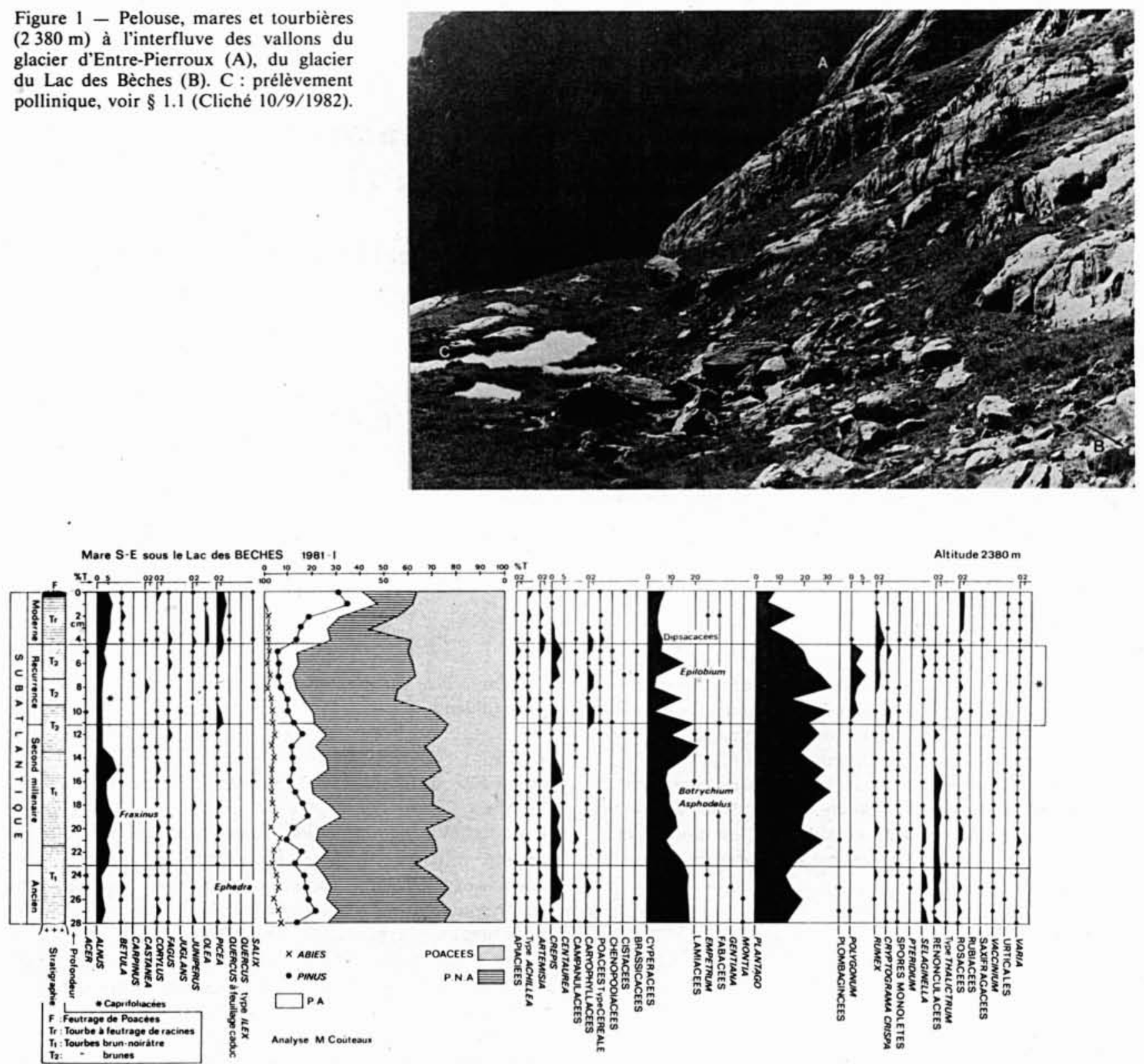

Altitude $2380 \mathrm{~m}$

Figure 2 - Diagramme pollinique de la mare sous le Lac des Bèches.

Figure 3 - Lac des Rouies (2722 m). A : glacier de La Lavey, couvert, B : exutoire, C : cordons morainiques des $17^{e}-19^{e}$ siècles, D : terrasse de Kame des $17^{\circ}-19^{\circ}$ siècles, E : terrasse de kame subactuelle. En arrière-plan glacier inférieur est des Sellettes. Voir § 1.2 (Cliché 15/9/1982).

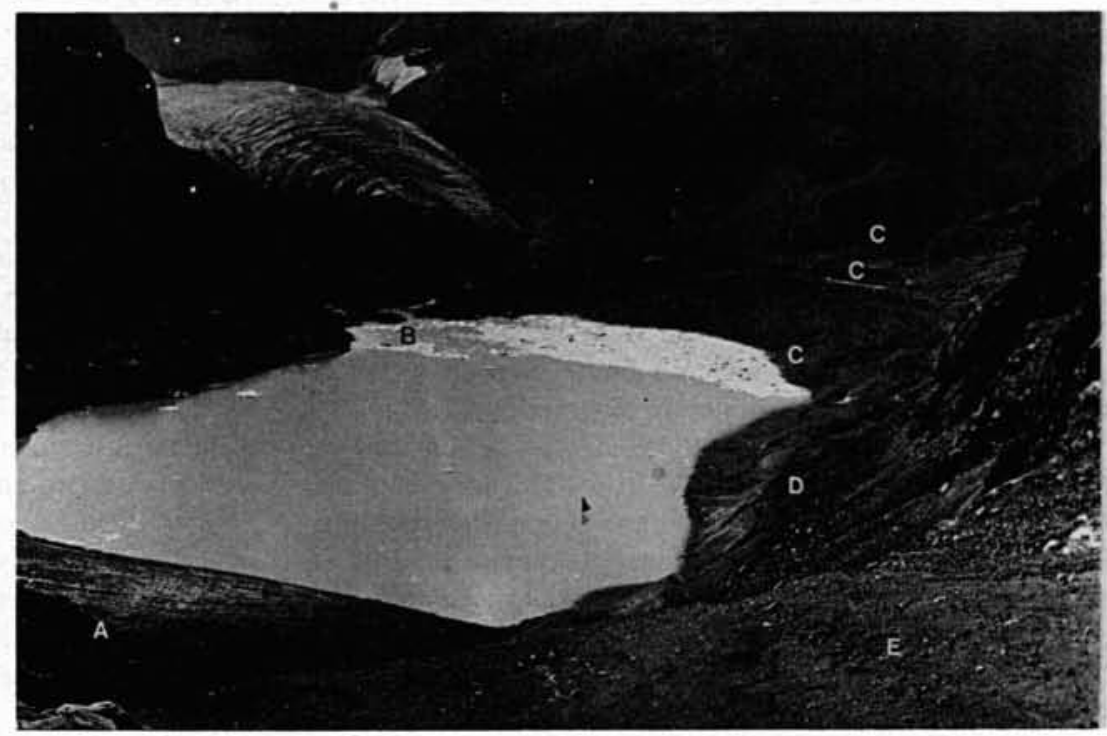




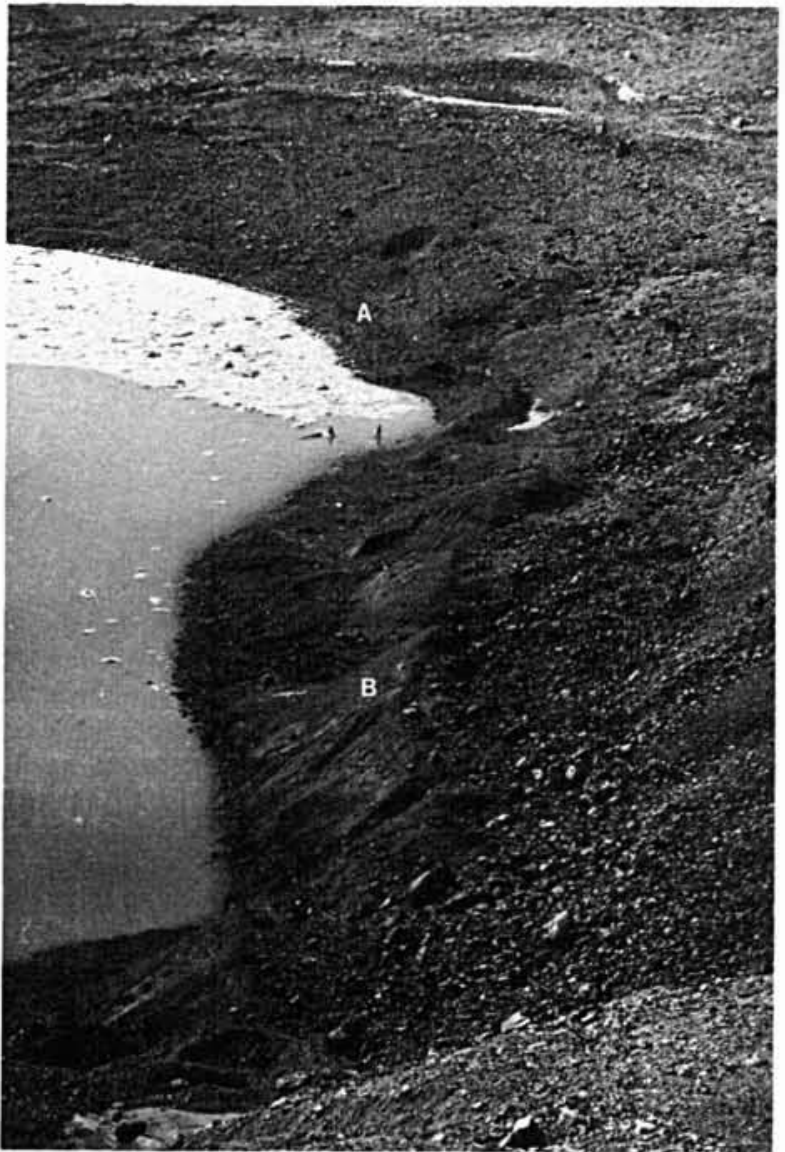

Figure 4 - Rive droite du Lac des Rouies. A : prélèvement pollinique dans la plage de Bryophytes, B : prélèvement pollinique sur le flanc de la terrasse de kame, voir $\$ 1.2$ (Cliché 15/9/1982).

La végétation de la tourbière semble affectée par les variations climatiques qui provoquent la récurrence glaciaire. On constate de brusques variations de fertilité des Cyperaceae, l'apparition d'une Caryophyllaceae et de Polygonum (cf. viviparum). Mais il est difficile de traduire ces variations de fréquences polliniques en termes phytosociologiques et donc d'en déduire un critère de différentiation de récurrence.

En conclusion, si la récurrence glaciaire des $17^{\circ}-19^{c}$ siècle est un phénomène à portée géomorphologique considérable, elle ne se manifeste que de façon très modeste dans les diagrammes de tourbes, seule la comparaison de nombreux sites à permis de la mettre en évidence.

\subsection{Lac des Rouies (fig. 3 et 4)}

Dans l'étage alpin, les conditions écologiques sont défavorables à l'édification de tourbières. La sédimentation minérale est parfois importante dans certaines zones affectées par les glaciers. Nous avons recherché les caractéristiques polliniques des dépôts morainiques du glacier de La Lavey, contemporains de la dernière récurrence et postérieur au déglacement local (un peu avant 1930).

\subsubsection{Le site du diagramme 1980-I (fig. 5)}

A l'ouest du lac, à environ $300 \mathrm{~m}$ du front actuel du glacier de La Lavey $(2725 \mathrm{~m})$, entre les cordons morainiques frontaux les plus internes de la dernière récurrence glaciaire, une dépression sableuse d'une dizaine de $\mathrm{m}^{2}$ est étudiée.

La mousse Pohlia filium (Schimp.) Mart. recouvre toute la surface avec de rares Pogonatum urnigerum (Hedw.) P.B. et Bryum sp. (dét. J.P. Hébrard).

A l'emplacement du profil, au sein des bryophytes, Sagina linnaei Presl., Arenaria serpyllifolia L., Saxifraga bryoides L., Poa alpina L., Ranunculus glacialis L., Hutchinsia alpina $\mathrm{R}$. Br., Arabis alpina L., Epilobium alpinum L., Sisymbrium pinnatifidum DC., et Veronica alpina L. occupent chacun à peine 1 à $2 \%$ de la surface.

Seul les horizons supérieurs du profil, humifères, sont riches en pollen (de 0 à $5 \mathrm{~cm}$ ).

Ceci s'explique par le développement de la végétation pionnière qui non seulement capte le pollen (bryophytes) mais en produit localement (bien qu'en quantités modestes). Ce sol est situé dans des conditions favorables à l'étude de la pluie pollinique en zone de retrait glaciaire en situation proxi-glaciaire. Les sédiments inférieurs (de 6 à $20 \mathrm{~cm}$ ) témoignent du mode de sédimentation pollinique en conditions glaciaires, ils sont très pauvres en pollens.

Tous les spectres polliniques de l'ensemble du profil sont caractérisés par une absence totale de corrélation entre les fréquences polliniques et les taux de recouvrement des végétaux localement présents.

Cette distorsion des spectres polliniques est avant tout la conséquence de l'extrême rareté des producteurs polliniques locaux, rareté due non seulement au caractère pionnier de la végétation, mais encore à l'altitude élevée du site étudié et surtout à la proximité de vastes surfaces dépourvues de végétation vasculaire (lac, glaciers, névés).

Les distorsions sont particulièrement remarquables au niveau de Pinus, de Sagina et d'Artemisia.

\section{a - Pinus}

Il n'existe actuellement aucun Pinus sur le pourtour du lac des Rouies. Or les fréquences polliniques dépassent toujours $40 \%$, atteignant même à $6 \mathrm{~cm}$ une valeur caractéristique en basse altitude de milieux forestiers : $93 \%$. Plus le sédiment est pauvre en pollen, plus les fréquences relatives de Pinus s'élèvent. Il est donc clair que l'apport allochtone de pollen de Pinus se perçoit d'autant mieux qu'il n'y a pas, ou peu, de producteurs polliniques locaux, c'est-à-dire que les conditions sont de plus en plus glaciaires. Il en va de même pour les autres pollens arboréens, quoique de façon plus discrète.

Lorsque l'" amélioration climatique " permet le développement d'une végétation locale, les fréquences polliniques des Pinus diminuent.

\section{b - Sagina}

Dans la partie supérieure du profil, une augmentation considérable des fréquences polliniques d'une Caryophyllaceae de type Sagina (Det. M. Reille), témoigne de l'installation locale de la végétation. Le producteur est local mais la sur-représentation est considérable : même les horizons humifères du profil contiennent donc des 

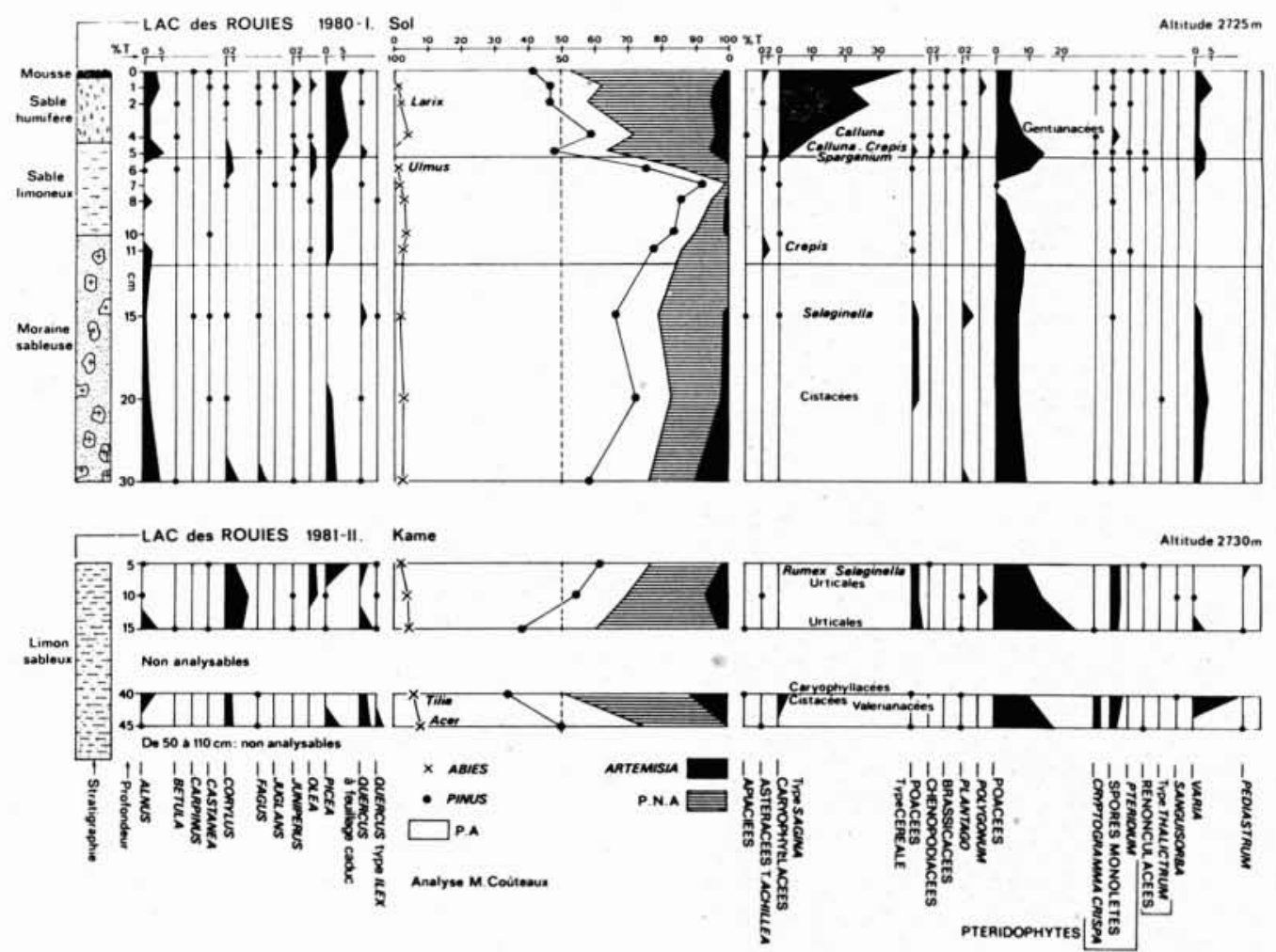

Figure 5 - Diagramme pollinique du Lac des Rouies.

Figure 6 - Zone proglaciaire du glacier du Lac des Bèches. A, B, C : moraines frontales de la récurrence des $17^{\mathrm{e}}-19^{\mathrm{e}}$ siècles, D : Lac des Bèches, E : prélèvement pollinique, voir $\S 1.3$ (Cliché 10/9/1982).
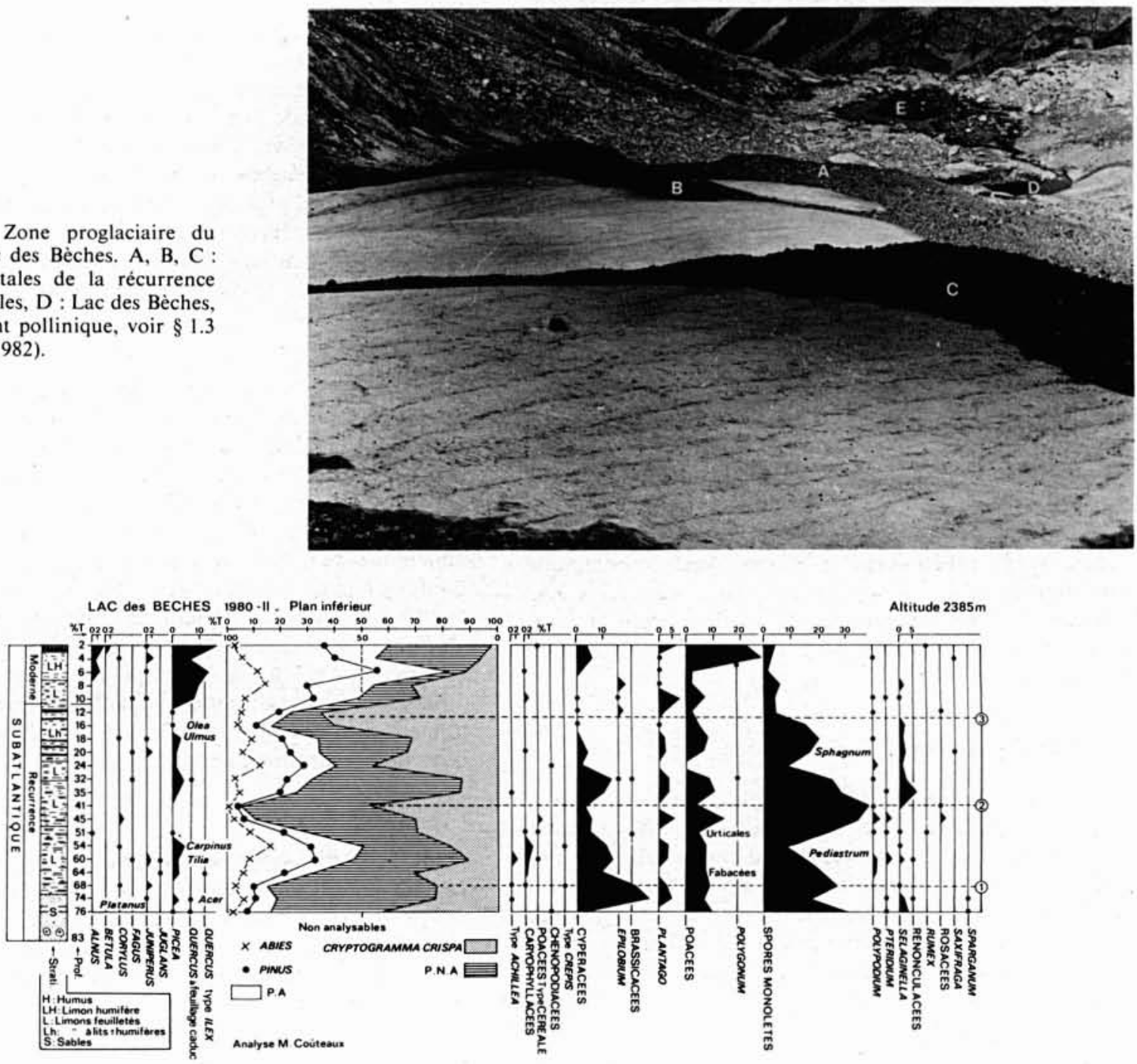

Figure 7 - Diagramme pollinique du plan inférieur du Lac des Bèches. 
ensembles polliniques abérants; au vu du diagramme pollinique, il est impossible de se faire une idée de la densité de la couverture végétale et de sa composition, d'autres pollens non arboréens étant eux-aussi allochtones (céréales, Cryptogramma crispa p. ex.).

\section{c - Artemisia}

A l'exclusion de la surface, les cinq centimètres supérieurs du profil sont caractérisés par des taux d'environ $5 \%$ d'Artemisia. Aucune espèce du genre ne figure dans le relevé de la végétation locale actuelle mais Artemisia umbelliformis Lam. est fréquente dans les environs proches, parfois accompagnée par $A$. genipi Web. et $A$. eriantha Ten. Tout permet donc de croire que l'augmentation des fréquences polliniques d'Artemisia observée à $5 \mathrm{~cm}$ témoigne de l'extension des groupements à Artemisia dans les zones récemment déglacées en bonne conformité avec l'existence de ces plantes sur des épandages glaciaires très récents, par exemple en rive droite du lac. Mais les pollens d'Artemisia font partie du flux pollinique allochtone : le recul des fréquences observé de 1 à $0 \mathrm{~cm}$ est un artefact du à la plus grande production pollinique locale de Sagina. Les fluctuations de la courbe d'Artemisia ne peuvent donc être parallélisées avec les fluctuations des groupements végétaux à Artemisia; il faut admettre qu'elles traduisent indirectement les fluctuations locales du glacier, les plus grandes fréquences (1 à $5 \mathrm{~cm}$ et $30 \mathrm{~cm}$ ) sont caractéristiques non de conditions glaciaires mais de conditions proxi-glaciaires. Il est évident que lorsque le glacier s'éloigne, l'augmentation de la densité de la végétation locale permet à d'autres bon producteurs polliniques de diversifier les ensembles polliniques et d'effacer petit à petit l'influence des pollens allochtones, même si, près du glacier, les surfaces occupées par Artemisia sont en extension.

Les distorsions des spectres polliniques provoquées par la supra-représentation locale (Sagina) ou les apports allochtones (Artemisia et Pinus) rendent impossible l'utilisation des analyses polliniques pour décrire l'évolution locale de la végétation. Mais elles permettent de caractériser soit le caractère glaciaire d'un sédiment (de 20 à $5 \mathrm{~cm}$ ), soit son caractère proxi-glaciaire $(30 \mathrm{~cm}$ et de 5 à $0 \mathrm{~cm})$. Une partie du flux pollinique allochtone provient de l'étage alpin des zones non englacées. Mais l'apport principal provient de la végétation forestière des étages inférieurs, favorisant la datation des sédiments grâce à la présence de certains taxons sans qu'il soit possible de trouver aucun parallélisme dans l'évolution des fréquences polliniques, au contraire (Pinus).

\subsubsection{Le site du diagramme 1980-II (fig. 5)}

En rive droite du lac (nord), une terrasse de kame (Boulton et Eyles, 1979) édifiée sur le flanc droit du glacier durant la dernière récurrence, contient vers $2730 \mathrm{~m}$ un banc limoneux. De 110 à $50 \mathrm{~cm}$ le sédiment est quasi stérile donc glaciaire. De 43 à $5 \mathrm{~cm}$, cinq spectres attestent de l'existence, localement ou dans le voisinage, de steppes alpines à Artemisia avec un flux pollinique forestier allochtone important. L'analogie avec les spectres proxiglaciaires du diagramme 1980-I est évidente.
Certains niveaux, trop pauvres pour être analysés, contiennent des pollens remaniés de sédiments antérieurs, probablement d'anciennes moraines démantelées. Tel est le cas du niveau 20 où il y a Tilia et plus d'Abies que de Pinus. Le comptage obstiné de tels niveaux selon les concepts des pollenanalystes archéologues français, aurait conduit à construire un diagramme absurde.

\subsubsection{Conclusions des analyses du lac des Rouies}

Le caractère glaciaire des sédiments minéraux est pollenanalytiquement caractérisé dans l'étage alpin, soit par l'absence ou la très grande rarèté de pollen, soit par la présence de pollens remaniés donnant des spectres incohérents entre eux, soit par des apports polliniques allochtones principalement forestiers.

Le caractère proxi-glaciaire des sédiments minéraux est pollenanalytiquement caractérisé par une diminution du flux pollinique forestier allochtone grâce à la présence de producteurs polliniques locaux du voisinage. Artemisia, supra-représentée, s'avère la meiHeure caractéristique des milieux proxi-glaciaires, les espèces alpines de ce genre étant largement répandues et bonnes productrices polliniques.

Dans les sédiments pauvres en pollens de l'étape alpin, un maximum de Pinus peut être contemporain d'un minimum de Pinus dans une tourbe de l'étage subalpin.

\subsection{Plan inférieur du Lac des Bèches (fig. 6)}

En contrebas de l'exutoire du lac, à $2385 \mathrm{~m}$, soit à une altitude quasi identique à celle de la tourbière (fig. 2), mais cette fois-ci en aval du glacier du Lac des Bèches, à $850 \mathrm{~m}$ du front actuel du glacier du Lac des Bèches, une petite plaine de type "sandur " est colmatée par des alluvions silto-sableuses proglaciaires de la dernière récurrence, actuellement stabilisées par une pelouse d'installation récente, mise en évidence dans les niveaux superficiels du diagramme pollinique (fig. 7) par l'extension des Poaceae. L'écho classique du reboisement récent de la vallée est perçu dans les spectres supérieurs dès $10 \mathrm{~cm}$ (Picea. Pinus). Le milieu de dépôt et les environs n'étant pas totalement dépourvus de végétation, la distorsion des courbes des taxons forestiers est moins forte qu'en position proglaciaire aux altitudes plus élevées de l'étage alpin.

Le phénomène caractéristique réside dans l'existence de trois phases (fig. $7: 1,2,3$ ) durant lesquelles les spores de Ptéridophytes, principalement Cryptogramma crispa. sont tellement abondantes que leur supra-représentation efface la supra-représentation des taxons forestiers. L'interprétation du phénomène doit faire appel à un enneigement considérable de l'étage subalpin durant trois sousstades de la dernière récurrence. La vitalité des végétaux locaux est totalement inhibée sauf sur certains éboulis d'adret oủ les Ptéridophytes peuvent encore murir leurs spores à la fin de l'été. Le flux pollinique forestier remontant n'a aucune raison d'avoir diminué durant les périodes à enneigement prolongé; il est probable que le dépôt printanier des pollens se faisait sur la neige encore épaisse et qu'il a été éliminé au début du dégel sans pouvoir se mêler à la sédimentation minérale. 
A La Lavey, actuellement, les formations détritiques de pente riches en Cryptogramma crispa (grosse productrice de spores à faible dispersion) sont fréquentes, où l'on constate un retard de sporaison en fonction du déneigement plus ou moins tardif.

Le diagramme met en évidence trois phases à nivation optimale. Dans le massif du Mont-Blanc, Vivian (1975) a daté par des documents historiques, plusieurs pulsations glaciaires durant la récurrence subatlantique, qui ont été corrélées en Vanoise par Tessier (1981) avec des périodes de faible croissance de Larix : les recherches dendroclimatologiques ont prouvé que l'enneigement printanier prolongé et les précipitations estivales élevées étaient les causes climatiques du phénomène.

En conclusion, Cryptogramma crispa a dans les sédiments minéraux de l'étage subalpin une importance pollenanalytique particulière, puisqu'elle souligne par son abondance les phases à enneigement optimum. De même que dans l'étage alpin Artemisia avait une signification proxi-glaciaire, on dira que Cryptogramma a dans l'étage subalpin une signification proxi-nivale.

La comparaison des deux diagrammes du lac des Bèches (fig. 2 et fig. 7) permet de proposer une interprétation climatique des trois phases caractérisées dans la tourbe, lors de la dernière récurrence, par des valeurs basses des fréquences polliniques des Cyperaceae ainsi que par la présence de quelques pollens de Caryophyllaceae et de rares spores de Cryptogramma crispa: il s'agirait d'une manifestation discrète des trois sous-stades à enneigement optimal.

\section{La récurrence glaciaire du Dryas supérieur}

Dans l'étage alpin (La Muzelle à Venosc), plusieurs diagrammes polliniques récemment publiés (Coûteaux, $1982 \mathrm{a}$ et b, $1983 \mathrm{a}$ ) datent une récurrence glaciaire locale du Dryas supérieur en synchronisant des sédiments lacustres proglaciaires et des moraines. Au même moment, dans l'étape subalpin (Les Deux-Alpes), plusieurs diagrammes polliniques récemment publiés (Coûteaux et Evin, 1981, Coûteaux, 1983 b) datent une phase de nivation. Dans les deux cas, trois pulsations sont mises en évidence au sein de la récurrence, soulignées par la courbe d'Artemisia affectée positivement par la proximité glaciaire, ou par la courbe de Selaginella affectée positivement par la couverture nivale.

\subsection{La Muzelle}

A la Muzelle (2 $140 \mathrm{~m}$ ), le site étudié est au Dryas inférieur un lac proglaciaire; des argiles varvées y sédimentent dans lesquelles la proportion des pollens d'Artemisia est élevée. Du Bølling au début du Dryas supérieur, les argiles ne sont plus varvées car le lac n'est plus alimenté par les eaux issues du glacier en recul. Au Dryas supérieur, une récurrence glaciaire met en place un complexe morainique et permet à nouveau le dépôt d'argiles varvées.
Depuis le début du Tardiglaciaire, la végétation a profondément évolué, les steppes à Artemisia sont colonisées au Bølling par une fruticée à Juniperus, remplacées à l'Allerød par une forêt de Pinus qui est intensément dégradée durant le Dryas supérieur alors que s'observent à nouveau des fréquences significatives d'Artemisia.

Durant l'Holocène, le glacier s'éloigne de plus en plus du site étudié: les fréquences polliniques d'Artemisia deviennent négligeables.

Lorsque le glacier affecte la sédimentation lacustre, les fréquences polliniques d'Artemisia sont élevées. Lorsque le glacier est éloigné du milieu de dépôt du pollen, elles sont faibles. Ici encore il est donc permis de lier les fréquences élevées d'Artemisia à la proximité glaciaire.

Deux particularités de la courbe d'Artemisia permettent d'affiner l'analyse des conditions de milieu tardiglaciaires du site de La Muzelle.

1) Dès la fin du Dryas inférieur, les fréquences polliniques diminuent. Ce phénomène se poursuit durant le Bølling et le Dryas moyen : rien ne permet donc de parler d'avancée glaciaire ou Dryas moyen. Par contre, à la charnière entre le Dryas moyen et l'Allerød, lorsque l'augmentation des fréquences polliniques de Pinus indique déjà le début de l'installation forestière, une pointe d'Artemisia pourrait témoigner d'une légère récurrence glaciaire, hypothétique.

2) Durant le Dryas supérieur, les diagrammes indiquent trois maximums d'Artemisia, il y aurait donc eu trois sous-stades, le second accompagné de l'avancée glaciaire la plus étendue, car c'est alors que la sédimentation lacustre est à nouveau varvée.

En conclusion, les déductions tirées du comportement d'Artemisia a La Muzelle durant le Tardiglaciaire se recoupent avec les observations faites sur les sédiments subatlantiques des lacs des Rouies et des Bèches qui prouvent les liens entre les fréquences polliniques d'Artemisia et les distances aux glaciers. Il ne faut pas perdre de vue que l'interprétation écologique de la courbe d'Artemisia est complexe. Actuellement, en Oisans, les groupements à Artemisia sont fréquents dans l'étage alpin à proximité immédiate ou en surplomb des glaciers. Ils se développent rapidement sur les épandages morainiques modernes. A priori, on devrait donc les considérer comme témoins du recul glaciaire. En fait, l'installation d'une végétation diversifiée à la suite du recul glaciaire conduit rapidement à un recul de la fréquence pollinique relative d'Artemisia au profit de la fréquence du pollen des autres taxons colonisateurs ( Poaceae, etc.). Au contraire, quand le glacier et l'enneigement sont fort étendus, les taxons persistants sur les nunataks sont les seuls producteurs polliniques en altitude et, parmi eux, Artemisia est bon producteur. Il y a donc dans ces conditions une suprareprésentation d'Artemisia. Les fréquences temporairement plus élevées d'Artemisia témoignent donc effectivement d'une extension glaciaire sans pour autant témoigner d'une extension des groupements à Artemisia.

La comparaison des spectres du Dryas supérieur de La Muzelle (autour de 60 à $70 \%$ de pollen de Pinus) et des spectres proxi-glaciaires du Lac des Rouies montre à quel point à dû être intense la dégradation de la forêt alpestre au terme de l'Allerød, une grande partie du flux pollinique forestier du Dryas étant nécessairement allochtone. 


\subsection{Les Deux-Alpes}

Aux Deux-Alpes, les sédiments sont prélevés à plus faible altitude $(1646 \mathrm{~m})$ dans une ancienne auge de transfluence glaciaire Vénéon-Romanche. Le morcellement des glaciers dès le début du Tardiglaciaire force à considérer toute transfluence ou diffluence locale comme au moins pléniwürmienne. Au Tardiglaciaire, le fond de l'auge est donc libre de glace : dans le massif interfluve Vénéon-Romanche, les fronts du Dryas supérieur sont généralement observés au-dessus de $2000 \mathrm{~m}$. Or, l'examen des sédiments fluviatiles met en évidence d'incontestables concordances avec les phénomènes observés à La Muzelle. La courbe d'Artemisia n'est pas significative à cause de l'éloignement des glaciers, mais la courbe de Selaginella selaginoïdes présente trois maximums durant le Dryas supérieur ainsi qu'une petite poussée durant le début de l'Allerød.

Durant le second maximum de Selaginella, une phase d'érosion des sédiments antérieurs est à l'origine d'un système de chenaux ultérieurement comblés par des graviers. A l'emplacement des profils analysés, la sédimentation est de 5 à 17 fois plus importante durant l'ensemble du Dryas supérieur que durant la période, trois fois plus longue, qui va du Bolling à l'Allerød. Le plan des Deux-Alpes est donc témoin d'une faible activité fluviatile durant le Tardiglaciaire sauf au Dryas supérieur caractérisé par de profondes modifications, alors même que la courbe de Selaginella est affectée de trois fluctuations analogues aux trois fluctuations de la courbe d'Artemisia à La Muzelle. Dans les deux sites, la deuxième fluctuation est synchrone du changement significatif du rythme sédimentaire.

La synchronisation des phénomènes sédimentaires observés à la Muzelle et aux Deux-Alpes ne pose guère de problèmes (Coûteaux, 1982c) mais l'interprétation écologique des fluctuations de la courbe de Selaginella est malaisée.

Aucun diagramme pollinique des Alpes françaises ne contient des taux aussi élevés de spores de Selaginella durant le Tardiglaciaire (Beaulieu, 1977; Wegmüller, 1977).

Ailleurs, les fréquences élevées sont très rares et n'ont pas été expliquées : dans les Carpathes polonaises (Ralska - Josiewiczowa, 1980), des sédiments de l'Allerød contiennent 10 à $20 \%$ de Selaginella; durant le Pléni-Würm, des sédiments de Yougoslavie (Sercelj, 1967) contiennent des fréquences de l'ordre de $25 \%$.

Les comparaisons avec les conditions actuelles manquent. Ce taxon s'est probablement maintenu quasi seul dans des zones humides localisées, lorsqu'un enneigement local exceptionnel inhibait le développement de la végétation tardiglaciaire. Il y a donc une supra-représentation des spores de Selaginella dans les alluvions, ne témoignant pas d'une extension de groupements végétaux à Selaginella, mais liés à l'extension nivale. Les recherches sur la distribution actuelle de Selaginella selaginoides en Oisans nous ont permis de noter l'abondance de l'espèce dans l'étage subalpin et sa quasi-absence dans l'étage alpin : il en était probablement déjà ainsi au Tardiglaciaire et le phénomène ne peut pas être décelé à La Muzelle. Mais, on ne retrouve pas aux Deux-Alpes des fréquences significatives de Cryptogramma crispa durant les phases de nivation, car les calcschistes du Lias ne donnent que de petits congelifracts et des sols argileux : les formations détritiques grossières manquent ici.

En conclusion, au même titre que Cryptogramma crispa, les fréquences de Selaginella traduisent des fluctuations de la couverture nivale en conditions périglaciaires et donc renforcent les déductions climatiques tirées des courbes d'Artemisia en conditions glaciaires.

La comparaison des sites de La Muzelle et des Deux-Alpes force à admettre que les analogies observées, correspondant à des conditions écologiques différentes, doivent être mises en relation avec des facteurs climatiques analogues.

La déglaciation de l'étage alpin et le déneigement de l'étage subalpin sont, en Oisans, importants durant le Tardiglaciaire, sauf durant le Dryas supérieur caractérisé par une récurrence stadiale à trois sous-stades. Paradoxalement, les "stades" d'avancée glaciaire ne datent pas systématiquement des périodes présumées "stadiales ": il n'y a aucune trace d'avancée glaciaire durant le Dryas inférieur et le Dryas moyen, au contraire. Le système proposé par Hannss (1973 et 1978) n'est donc pas applicable à l'Oisans. Des systèmes de Beaulieu et Jorda (1977) et de Francou (1981), il faut retenir comme probablement bien daté du Dryas supérieur le "stade III de Molières " et le " stade du Lautaret ", mais rejeter dans le Pléni-Würm, les stades antérieurs.

\section{La déglaciation du Dryas inférieur}

Un des phénomènes observés au lac des Rouies (diminution de la supra-représentation de Pinus et augmentation des fréquences polliniques d'Artemisia) est comparable à ce qui a été noté dans l'Ain, le Velay, le Cantal (Beaulieu et al. 1983) au début du Tardiglaciaire, lors de l'extension des steppes. Vers $15000 \mathrm{BP}$, au début de l'amélioration climatique du Tardiglaciaire, le développement de la végétation steppique locale conduit à une régression de la proportion relative du pollen allochtone de Pinus. Cette interprétation est donc vérifiée par les analyses du lac des Rouies qui permettent en outre de préciser que les fréquences polliniques des taxons steppiques sont ellesmêmes supra-représentées: le recouvrement réel des plantes vasculaires était certainement très lâche. Seul subsiste le problème de la datation du phénomène. Dans le cas du Lac des Rouies, les pollens des essences forestières tempérées indiquent un âge subatlantique récent. Puisque le recul des fréquences polliniques de Pinus est lié au recul local du glacier, le phénomène en soi n'a pas d'âge et pourrait être noté à n'importe quelle époque de l'Holocène, datable par le flux allochtone. Durant le Tardiglaciaire, en absence des pollens forestiers tempérés, un recul de Pinus suivi d'une extension d'Artemisia n'est connu qu'à deux moments :

- après l'Allerød, il s'agit d'une réelle régression de Pinus et d'une réelle extension d'Artemisia vers $10900 \mathrm{BP}$, la datation de cette phase ne pose généralement pas de problème majeur; 


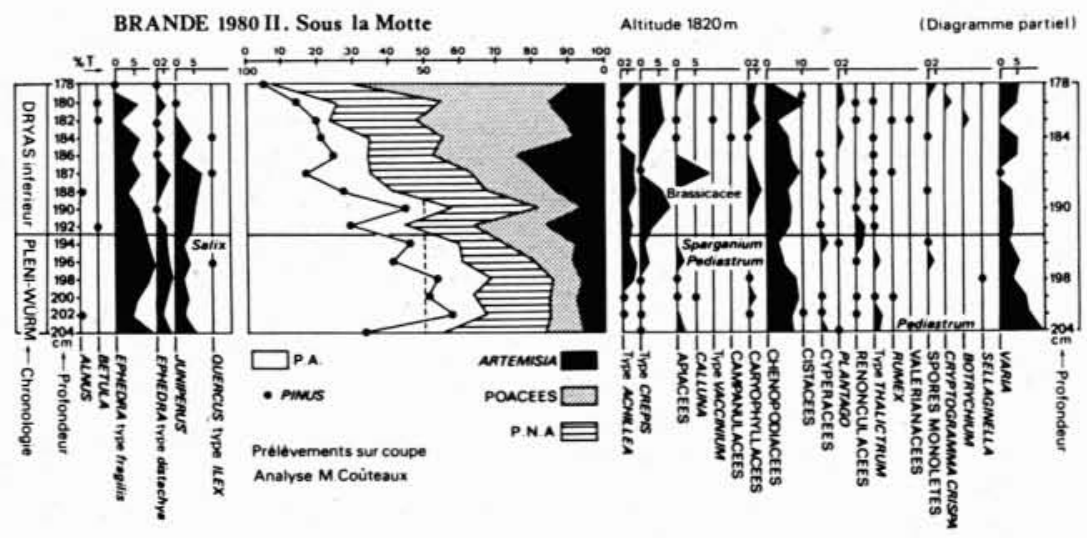

Figure 8 - Diagramme pollinique de Brande.

- à la charnière entre le Pléni-Würm et le Tardiglaciaire, vers $15000 \mathrm{BP}$, il s'agit alors d'un artefact du aux apports allochtones.

Un sédiment du début du Tardiglaciaire est connu en Oisans à $1820 \mathrm{~m}$ à l'Alpe d'Huez dans le site de Brande (Coûteaux, $1982 \mathrm{~d}$ ) (fig. 8) où, vers $194 \mathrm{~cm}$ de profondeur s'observe la chute des fréquences polliniques de Pinus allochtones et l'installation de la végétation steppique locale et de voisinage à Artemisia, Poaceae etc. Cette phase n'avait pu être datée auparavant faute de diagrammes de référence. Nous pouvons donc affirmer maintenant que le site de Brande était en voie de déglacement dès la fin du Pléni-Würm quoique situé dans l'axe de la vallée glaciaire de Combe Charbonnière. Les cordons morainiques visibles en amont entre 1900 et $2000 \mathrm{~m}$ dateraient ainsi de la grande récurrence du Dryas supérieur, en bonne conformité avec les datations des moraines de La Muzelle.
Par analogie avec les interprétations des fréquences polliniques d'Artemisia au Subatlantique et au Dryas supérieur, on déduit que les spectres riches en Artemisia de l'Alpe d'Huez témoignent de conditions proxi-glaciaires. Cette conclusion ne peut toutefois être appliquée que dans le contexte glaciaire alpestre. En effet, les steppes à Artemisia ont existé à plus basse altitude, loin de tout glacier; dans le nord de l'Europe, c'est le développement de ces steppes qui permet de dater les sédiments du début du Tardiglaciaire (Van Der Hammen, 1953) loin du front glaciaire. Cetaines parties du Massif Central sont restées à l'écart des glaciations würmiennes, comme le Velay (Veyret, 1981). Il faút donc en conclure que dans les régions non alpestres caractérisées par la rigueur du climat périglaciaire (Valadas, 1983), d'autres facteurs entrent en ligne de compte (enneigement, taxons différents) qui sont hors de propos ici.

\section{Conclusions}

\subsection{Ensembles polliniques caractéristiques}

Si le milieu de dépôt est couvert de glace ou de neige, les sédiments sont pratiquemment stériles en pollens. Quand le milieu de dépôt est exposé à la pluie pollinique, mais situé dans une zone dépourvue de producteurs polliniques locaux, les flux polliniques allochtones donnent des spectres à distorsions caractéristiques. $\mathrm{Si}$ le milieu glaciaire est situé en zone supra-forestière, le flux de pollens arboréens, mẽme quantitativement modeste, est considérable en fréquence relative. Ces informations nouvelles complètent les études antérieures (Coûteaux, 1981) sur les problèmes de représentation pollinique en altitude.

Dans l'étage alpin, les Artemisia sont pratiquemment les seuls bons producteurs de pollen, de plus leur supra-représentation est significativement affectée par les fluctuations glaciaires. Dans l'étage subalpin, certaines Ptéridophytes sont très bonnes productrices de spores et leur supra-représentation est semblablement affectée par des variations d'enneigement: Cryptogramma sur les éboulis secs, Selaginella sur des versants schisteux humides.

\subsection{Modifications caractéristiques de la végétation}

L'interprétation des diagrammes polliniques durant les récurrences glaciaires, en terme de végétation, est malaisée à cause des distorsions. Toutefois, il ressort qu'en Oisans dans le Dryas supérieur, le phénomène majeur est une dégradation profonde de la forêt et, durant les $17^{\mathrm{c}}-19^{\mathrm{e}}$ siècles, une inhibition de certaines fonctions biologiques de Pinus. 


\subsection{Récurrences majeures}

Il ressort des recherches polliniques et morphologiques (Coûteaux, 1983 d) que durant le Tardiglaciaire et l'Holocène, seules les récurrences glaciaires du Dryas supérieur et des $17^{\mathrm{c}}-19^{\mathrm{e}}$ siècles ont une importance significative durant quinze millénaires de retrait glaciaire.

La récurrence glaciaire du Dryas supérieur apparaît clairement dans tous les diagrammes d'altitude de l'arc alpin et les moraines qui lui ont été attribuées sont nombreuses, principalement en Suisse et en Autriche. Des cartographies détaillées existent. Mais la nomenclature est pleine d'ambiguité, l'utilisation de la nomenclature de Penck et Bruckner (1909) par la plupart des auteurs impose l'intercalation de stades locaux supplémentaires : force est de reconnaître que l'utilisation du critère " ligne de neige " comme critère de datation absolue est responsable de toutes les contradictions qui existent dans la littérature, sauf quand il y a datation par le biais de la palynologie (Maisch, 1981). Les recherches entreprises en Oisans confirment l'âge Dryas supérieur de ce que la plupart des auteurs appellent le "stade de Egesen ", et font apparaitre des phénomènes mal connus : importance des phases de recul, existence de récurrence impossibles à déceler morphologiquement, par exemple au début de l'Allerød et, en certains sites, le premier sous-stade du Dryas supérieur. L'existence de trois sous-stades au sein de la récurrence du Dryas supérieur est en bonne concordance avec les observations géomorphologiques de Kerschner et al. (1981) et de Bortenschlager (1982) en Autriche.

La récurrence glaciaire des $17^{\mathrm{e}}-19^{\mathrm{e}}$ siècles est bien connue historiquement et morphologiquement (Edouard, 1977; Vivian, 1975), mais toute documentation pollinique correspondante manquait.

\subsection{Incidences climatiques}

Les conclusions climatiques qui résultent des recherches en Oisans imposent une grande simplification par rapport aux systèmes complexes proposés par la plupart des auteurs étudiant l'arc alpin. Il n'est évidemment pas question de nier que des fluctuations climatiques ont induit d'autres mouvements glaciaires, mais l'échelle des différents phénomènes est fondamentalement différente. L'ensemble du Tardiglaciaire et de l'Holocène apparaît donc comme une longue période à climat favorable au recul glaciaire, sauf durant environ huit siècles radiocarbone au Tardiglaciaire et environ trois siècles à l'Holocène. Brèves mais considérables, ces deux réavancées sont provoquées par une combinaison d'enneigement printanier prolongé, de précipitations estivales élevées et de températures surtout estivales basses. Il convient d'insister sur les facteurs enneigement-précipitations, bien mis en évidence par les recherches dendroclimatologiques pour la récurrence subatlantique (Tessier, 1981), mais qui ont dû jouer également un rôle essentiel dans l'alimentation des glaciers du Dryas supérieur. La plupart des auteurs insistent sur le caractère sec de cette dernière période, alors que l'importance de la récurrence sur l'ensemble de l'arc alpin prouve en soi la nécessité d'une augmentation considérable des précipitations efficaces. En outre, nous avons montré la péjoration de l'enneigement dans les zones périglaciaires et force est de reconnaître que les arguments botaniques généralement évoqués en faveur de la sécheresse peuvent être battus en brèche: Hippophae existe en régions humides, Artemisia n'est pas nécessairement en expansion quand ses fréquences polliniques augmentent, on ignore tout de l'interprétation des Chenopodiaceae, etc.

Le caractère humide du Dryas supérieur est mis en évidence en Autriche aux environs d'Innsbruck, incitant Kerschner et al. (1981) à faire entrer cette zone du Tyrol dans la partie humide des Alpes centrales durant le Dryas supérieur.

\subsection{Application de l'interprétation des distorsions}

Lorsque des sédiments de période pléni-glaciaires sont étudiés pollenanalytiquement, l'interprétation déjà malaisée dans les sédiments minéraux (Coûteaux, 1977) doit tenir compte des faits exposés. Or, les nombreuses publications de l'école française archéologique abondent en interprétations allant exactement à l'inverse de la réalité : la moindre augmentation des fréquences des courbes de Pinus est interprétée en terme de réchauffement. 


\section{Publications citées}

BEAULIEU J.L. de, 1977. - Contribution pollenanalytique à l'histoire tardiglaciaire et holocène de la végétation des Alpes méridionales françaises. Thèse ès sciences, Université Aix-Marseille III, 358 p., 29 fig., 39 diag. h.t.

BEAULIEU, CLERC, COUTEAUX, PONS et REILLE, 1983. Pollenanalyses and characters of climatic changes at the end of the Eemian and at the beginning of the Late-Würm in Western Europe. Paleoclimatic Research and models, Bruxelles Redel Edit. 109-113.

BEAULIEU J.L. de et JORDA M., 1977. - Tardiglaciaire et Postglaciaire des Alpes de Haute-Provence. Le Glaciaire de la Montagne de La Blanche - Trois Evéchés. Bull. $A F E Q$ 3, 3-15.

BORTENSCHLAGER S., 1982. - Chronostratigraphic subdivisions of the Holocene in the Alps. Striae, 16:75-79.

BOULTON G.S. et EYLES N., 1979. - Sedimentation by valley glaciers : a model and genetic classification. in Moraines and Varves, Rotterdam, 1979, II-23.

COUTEAUX M., 1977. - A propos de l'interprétation des analyses polliniques de sédiments minéraux principalement archéologiques. "Approche écologique de l'homme fossile ". Suppl. Bull. AFEQ, $47: 259-276$.

COUTEAUX M., 1981. - Caractérisation pollenanalytique en Oisans des milieux forestiers et des milieux supra-forestiers dans l'actuel et dans le passé. Actes du Colloque de Perpignan, avril 1981 : La limite supérieure de la forêt et sa valeur de seuil, p. 139-159.

COUTEAUX M., 1982 a. - Intérêt historique et scientifique de la tourbière de La Muzelle (commune de Venosc, Isère, France). Proposition en vue de sa protection. Actes du colloque de Chamonix : Montagne et Aménagement, Institut de Géographie Alpine, LAMA 1982, p. 77-83.

COUTEAUX M., 1982 b. - La tourbière et le glacier de La Muzelle (Parc National des Ecrins). Rev. Parc. Nat. Ecrins, 2, 31-52.

COUTEAUX M., 1982 c. - Récurrence glaciaires en Oisans au Tardiglaciaire. Soc. Hydr. Fr. Sec. Glacial. Actes de la réunion du 4-5 mars 1982, p. 1-3.

COUTEAUX M., $1982 \mathrm{~d}$. - Recherches pollenanalytiques en Oisans : le plateau de Brande (Alpe d'Huez, Isère, France); Bull. Soc. Roy. Bot. Belg. 115 (1) : 91-106.

COUTEAUX M., 1983 a. - Fluctuations glaciaire de la fin du Würm dans les Alpes françaises, établies par les analyses polliniques. Boreas, $12: 35-56$.

COUTEAUX M., 1983 b. - Géomorphologie et évolution phytogéographique tardiglaciaire et holocène aux Deux-Alpes (Isère, France) : contribution pollenanalytique. Rev. Géogr. alpine, 71 (2) : 143-163.

COUTEAUX M., 1983 c. - Déboisements d'altitude par les charbonniers de l'Oisans : contribution pollenanalytiques. $108^{\circ}$ Congr. Soc. Sav. Grenoble (sous presse).

COUTEAUX M., $1983 \mathrm{~d}$. - La déglaciation du vallon de La Lavey (vallée du Vénéon, Massif des Ecrins, Isère, France). Section de Glaciologie de la Société Hydrotechnique de France, comptes-rendus de la séance de Grenoble (mars 1983), p. 1-13.

COUTEAUX M., 1984. - Présence, datages et signification phytosociologique de macrorestes de Pinus et de pollens de
Pinus cembra L. à $2050 \mathrm{~m}$ dans le valon de La Lavey (Massif des Ecrins, Isère, France). Paleobiologie. Genève 55-62.

COUTEAUX M. et EVIN J., 1981. - Etudes palynologiques et datages par le radiocarbone de dépôts fluvioglaciaires aux Deux-Alpes (Isère, France). C.R. Acad. Sc., 292 sér. II : 1235-1238.

EDOUARD J.L., 1978. - La glaciation du Bassin de la Romanche. Contribution à l'étude des fluctuations glaciaires postwürmiennes. Thèse Grenoble, $329 \mathrm{p}$.

FRANCOU B., 1981. - Géodynamique des éboulis et formes associées de la Combe de Laurichard, Hautes-Alpes. Thèse $3^{\circ}$ cycle, $153 \mathrm{p}$. Grenoble IGA.

HANNSS C., 1973. - Conséquences morphologiques de nouvelles datations du $14 \mathrm{C}$ dans le sillon alpin près de Grenoble. Rev. Géogr. Alp., 51 : 179-201.

HANNSS C., 1978. - Formenschatz und Mutmassliches Alter einer spätglazialen Moräneabfolge im inneralpinen Trochengebiet der Vanoise (Französiche Nordalpen). Tub. Geogr. ST., 80, Festschrift Blume Trockengebiete : 177-193.

KERSCHNER H. et BERKTOLD E., 1981. - Spätglaziale Gletscherstände und Schuttformen im Senderstal, nördliche Stubaier Alpen, Tirol. Zeits. f. Gletsch. u. Glazialg. 17 (2) : 125-134.

MAISCH M., 1981. - Glazialmorphologische und gletschergeschichtliche Untersuchungen im Gebiet zwischen Landwasser - und Albulatal (Kt. Graubünden, Schweiz). Geogr. Inst. Univ. Zurich, 3, 217 p.

PENCK A. et BRÜCKNER E., 1909. - Die Alpen im Eiszeitalter. 3 t., XXVIII + 1199 p. Leipzig.

RALSKA-JOSIEWICZOWA M., 1980. - Late-glacial and Holocene vegetation of the Bieszczady mis. (Polish eastern Carpathians). Polks. Akad. N. Inst. Bot. 202 p.

SERCELJ A., 1967. - Die Entwicklung des Untergrundes um südlichen Teil von Ljubljana im Lichte der palynologischen Untersuchungen. Slov. Akad. Zuam. Um. Cl. IV, $10(7): 279-302$.

TESSIER L., 1981. - Contribution dendroclimatologique à la connaissance écologique du peuplement forestier des environs des chalets de l'Orgière (Parc National de la Vanoise). Trav. Sc. P.N.V. II, 19-61.

VALADAS B., 1983. - Les Hautes Terres du Massif Central français: Contribution à l'étude des morphodynamiques récentes sur versants cristallins et volcaniques. Thèse Paris I, 2 tomes, $927 \mathrm{p}$.

VAN DER HAMMEN T., 1953. - Late-Glacial flora and periglacial phenomena in the Netherlands. Leidse Geol. Med. 17, 17-84.

VEYRET Y., 1981. - Les modelés et formations d'origine glaciaire dans le Massif Central français: problèmes de distribution et de limites dans un milieu de moyenne montagne. Thèse Paris I, 2 tomes, 783 p.

VIVIAN R., 1975. - Les glaciers des Alpes occidentales. Etude géographique. Grenoble, Allier, 513 p.

WEGMUULLER S., 1977. - Pollenanalytische Untersuchungen zur spät - und postglazialen Vegetationsgeschichte der französischen Alpen (Dauphiné). Thèse, 185 p., Bern. 


\section{Discussion}

Président : M. L. de CRÉCY

M. le Président remercie Monsieur Cooteaux et ouvre la discussion en soulignant combien l'auteur insiste pour une interprétation prudente des diagrammes polliniques dans les zones de marges glaciaires.

M. COUTEAUX. - En effet, alors que les correspondances sont bonnes à basse altitude, les distorsions entre végétation et pluie pollinique s'accroissent avec l'altitude (diminution de la végétation).

M. LLIBOUTRY, - Pourquoi ne pas établir des diagrammes en valeur absolue?

M. CovteaUX. - C'est impossible si l'on ne connaît pas la durée d'une séquence ou le taux de sédimentation.

M. BUCCHER. - Avez-vous retrouvé des traceurs d'origine africaine liés aux poussières d'origine saharienne?

M. COUteaUX. - Non, mais cela ne veut pas dire qu'il n'en existe pas. Cela suppose une analyse pollinique plus poussée (cf. Laboratoire de Madame VAN CAMPO).

M. BEZINGE. - Vous êtes en retrait par rapport à certains de vos collègues suisses ou autrichiens qui relient directement les analyses palynologiques à l'histoire climatique. La prudence s'impose donc.

M. Covteaux. - Ce genre de raisonnement est fréquent dans la littérature, peut-être parce que la Palynologie est une science encore jeune.

$M^{\text {MU }}$ MUXART

1) Les pollens lointains ou anciens que vous retrouvez n'ont-ils pas un faciès plus abimé ?

2) Au sein de notre équipe, $\mathbf{M}^{\mathrm{me}} \mathrm{J}$. COHEN ayant soumis des échantillons de tourbière, en provenance du rebord SE du Massif Central, à deux traitements chimiques différents a pu observer - dans certains cas - des distorsions dans le spectre pollinique qui peuvent être considérables. Il convient de se montrer prudent dans l'interprétation des données.

M. CoUteaUX. - Non, il n'y a pas de raison. Le temps de transport peut être très court. S'il y a remaniement par ruissellement, le taux de corrosion peut-être élevé. Mais ce n'est pas parce qu'un pollen est plus abîmé qu'il est plus ancien. Les méthodes physiques favorisent les pollens à ballonnets. Les méthodes chimiques (qui détruisent les spores de sphaignes par exemple, ce qui est gênant à basse altitude), donnent des résultats différents. Vous avez raison d'insister là-dessus.

M. LLIBOUTRY. - Ne pourrait-on pas établir des gradients pour les pollens lointains?

M. COUTEAUX - En théorie oui; en pratique non, il faudrait, entre autres, connaître la végétation productrice locale.

M. POURCHET. - Les variations des taux de sédimentation pourraient peut-être être estimées en mesurant les variations de concentration d'un traceur émis avec un flux soit constant soit connu (radio-élément cosmogéniques par exemple).

M. COUTEAUX. - Ce travail devrait être entrepris avec d'autres laboratoires...

$M^{\text {mor }}$ EvIN. - Sur vos diagrammes polliniques, vous avez constaté trois phases avec diminution des P.A. interprétées comme des périodes plus glaciaires. Pensez-vous que cela soit dû à une diminution des températures ou une augmentation des précipitations?

M. COÔTEAUX. - Il y a corrélation entre ces trois phases du "Petit Age Glaciaire » et certains résultats quant à la croissance des cernes du mélèze en Vanoise, elle-même corrélée avec des facteurs climatiques. L'influence de l'humidité apparaît plus grande que la température (cf. thèse de L. TESSIER, ERA 404). Dans le milieu qui nous intéresse, on peut penser à un enneigement plus tardif, mais je n'ai pas de preuve. 


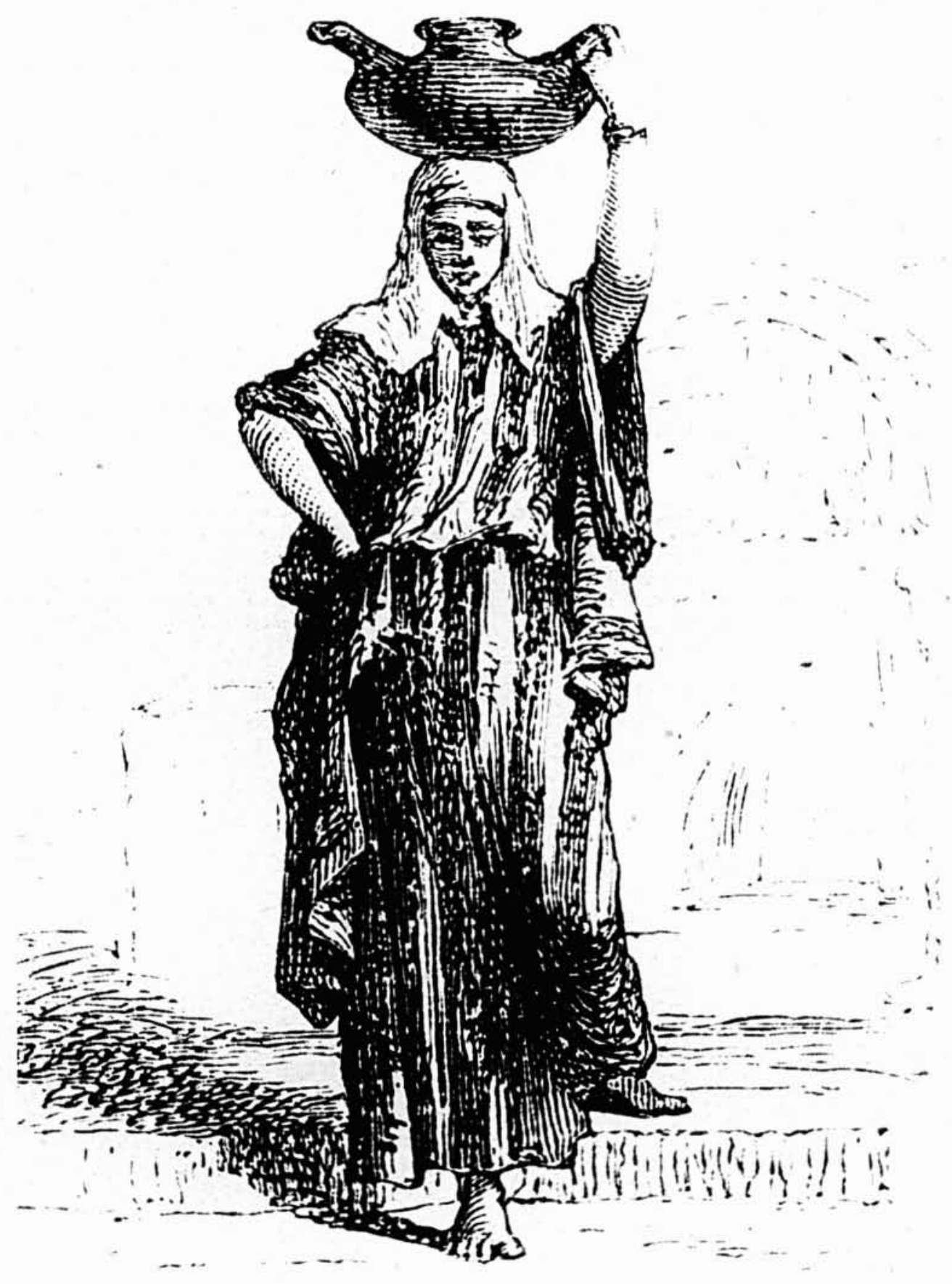

